\title{
CT Imaging in Predicting Ovarian Torsion: Report of Two Cases, With and Without Infarction
}

\author{
Sweta Singh $^{1}$, Prakash K. Sasmal ${ }^{2}$, Krishnan Nagarajan ${ }^{3}$ \\ 1. Obstetrics and Gyenacology, All India Institute of Medical Sciences, Bhubaneswar, IND 2. General Surgery, All India \\ Institute of Medical Sciences, Bhubaneswar, IND 3. Radiodiagnosis, Jawaharlal Institute of Postgraduate Medical \\ Education \& Research, Puducherry, IND
}

Corresponding author: Krishnan Nagarajan, lknagarajan1@gmail.com

\begin{abstract}
Ovarian torsion is one of the common abdominal and gynecologic surgical emergencies with serious morbidity in the form of ovarian infarct or necrosis. Various imaging modalities like USG, CT, and MRI have been used in the evaluation of ovarian torsion. Two middle-aged females presented with lower abdominal pain. Imaging showed an ovarian cystic lesion in the first patient and thickened and twisted ovarian pedicle or 'helical swirling' sign in the second. Intraoperatively, the first case turned out to be a 180 * twist with mild ovarian edema and the second showed more than $720^{*}$ torsion with ovarian infarction. The CT findings of twisted pedicle with pericystic fat stranding might be predictive of hemorrhagic infarction in cases of torsion.
\end{abstract}

Categories: Obstetrics/Gynecology, Radiology

Keywords: ovarian torsion, adnexal torsion, computed tomography, ovarian infarction, twisted pedicle

\section{Introduction}

The ovary is a female pelvic organ suspended in the pelvic peritoneal cavity by three peritoneal folds or ligaments - the infundibulopelvic fold or suspensory ligament, which attaches the ovary to the lateral pelvic wall, the utero-ovarian ligament connecting the ovaries to the uterus, and the mesovarium connecting the ovaries to the broad ligament of the uterus [1]. Torsion of an ovary, ovary with a tube, or rarely the tube alone can occur at any age. It is considered more common in children due to normal ligamentous laxity [2]. Ovarian torsion can occur with normal ovaries, ovaries having functional cysts, or with space-occupying lesions in the ovary such as dermoids (commonly) or other cystic or solid neoplasms [3, 4]. Torsion may impede the venous outflow causing edema and congestion. Later, the arterial inflow may be compromised ending in hemorrhagic infarction or necrosis. Imaging findings may vary depending on whether the torsion is partial or total, intermittent or progressive, and collateral supply of ovary from other vessels like the uterine artery branches [1].

Review began 07/04/2021 Review ended 08/11/2021 Published 08/11/2021

๑) Copyright 2021 Singh et al. This is an open access article distributed under the terms of the Creative Commons Attribution License CC-BY 4.0., which permits unrestricted use, distribution, and reproduction in any medium, provided the original author and source are credited.

\section{Case Presentation}

\section{Case 1}

A 48-year-old female presented with right lower abdominal pain for one month. She gave a history of a right adnexal cyst of small size. On USG, a multiloculated cystic lesion was noted in the right adnexa, which was relatively tender and had a mildly swollen appearance. The lesion showed normal surrounding vascularity with part of the normal ovary in continuity with the lesion. A CT scan confirmed the findings and did not show any surrounding fat stranding or fluid collection (Figure 1). 


\section{Cureus}

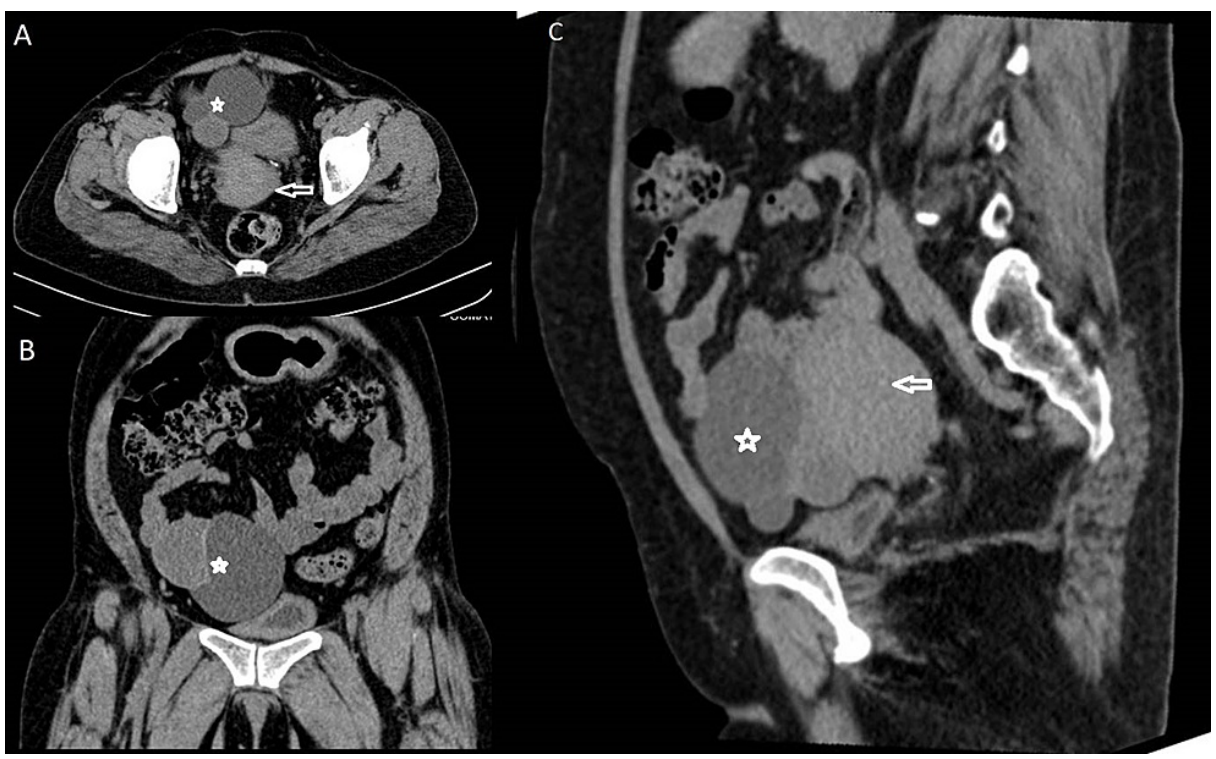

FIGURE 1: Case $1 \mathrm{CT}$ transverse section (A), coronal section (B), and sagittal section $(C)$ reformations showing multiloculated cystic lesion (marked with asterisks) anterolateral to the uterus (arrows in A \& C) on right side

No para-uterine or adnexal soft tissue thickening area was noted. On surgical exploration, a 180* twist of the cystic lesion and the ovary was seen (Figure 2). The ovary was grossly normal with only mild enlargement.
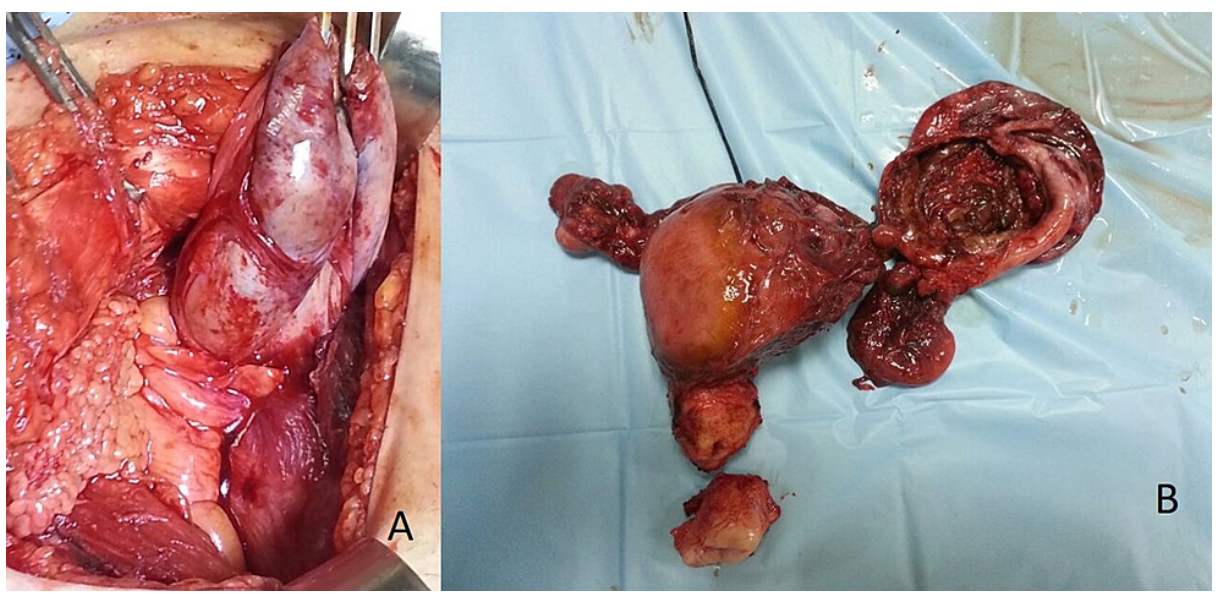

FIGURE 2: Case 1 (A) Intra-operative photograph and (B) gross specimen, showing partial twist and edematous cystic lesion (cut surface open in B) removed along with the uterus

\section{Case 2}

A 50-year-old female presented with a history of long-standing right lower abdominal discomfort that had increased since four days. She had moderate-to-severe right para-umbilical and lower abdominal pain with guarding. Ultrasonography revealed a large unilocular cystic lesion extending up into the para-umbilical region. The lesion showed increased peripheral vascularity and the right ovary was not separately visualized. The uterus and left ovary were normal. The cyst showed thick walls and subtle pericystic fat stranding, which are unusual for otherwise benign-looking features. There was helical soft tissue thickening that showed contrast enhancement extending from the lateral aspect of the uterus towards the cyst (Figure 3 and Figure 4). 


\section{Cureus}

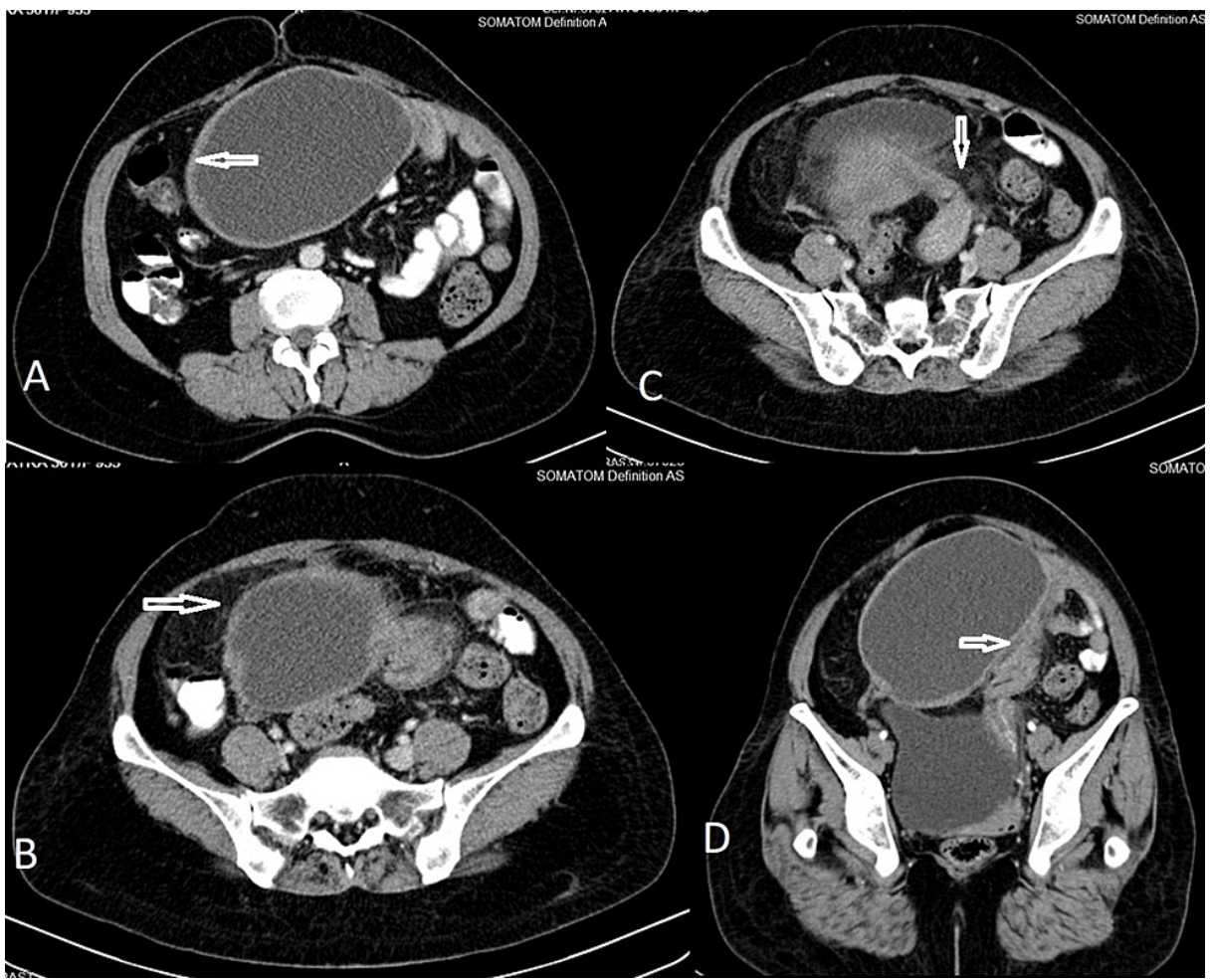

FIGURE 3: Case 2 CT axial sections (A, B, C) and oblique coronal reformation (D) showing a unilocular cystic lesion, anterosuperior to the uterus with thick walls (arrow in A), perilesional fat stranding (arrow in B), and twisted pedicle with 'helical swirl' appearance in continuous with left cornu of the uterus (arrows in C \& D)

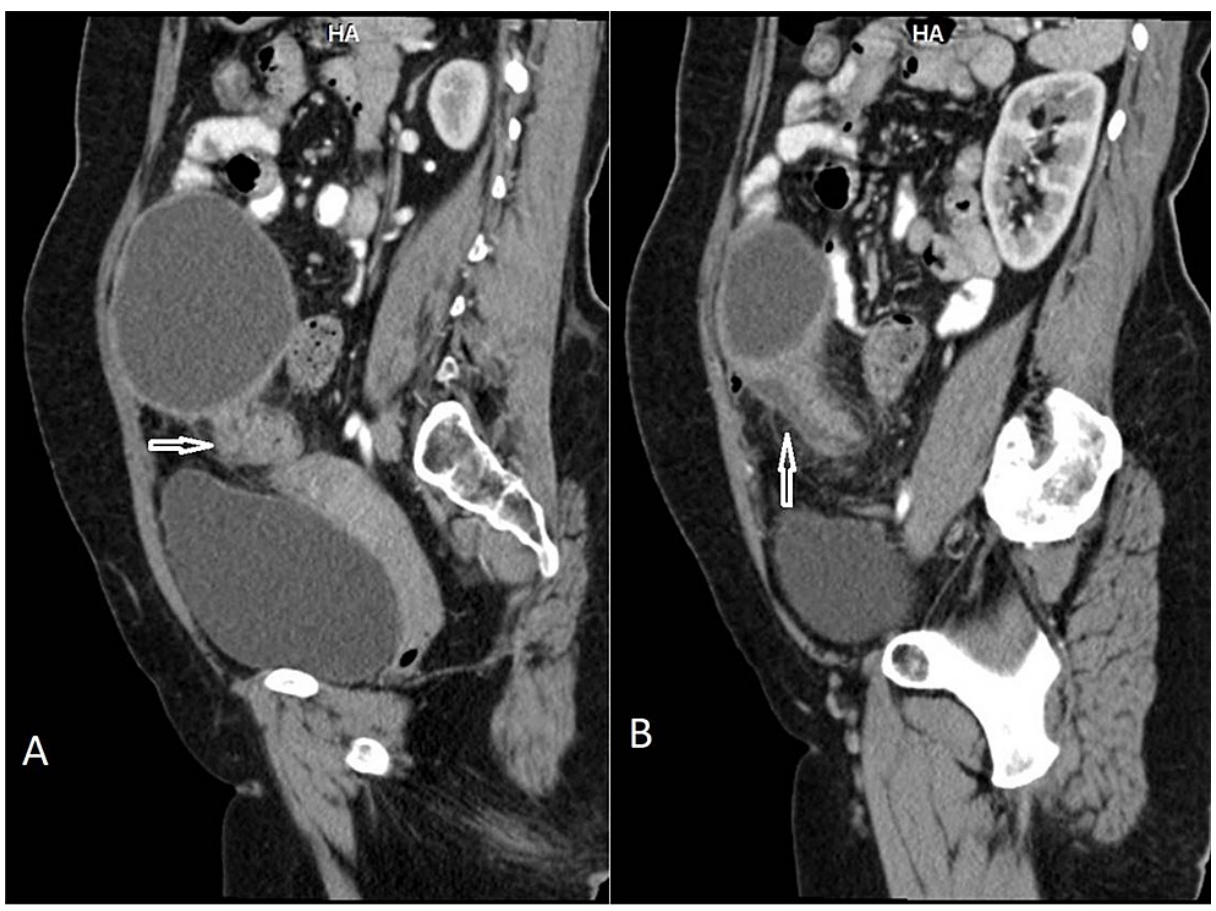

FIGURE 4: Case 2 CT sagittal reformations (A \& B) showing 'helical swirl' appearance of twisted pedicle between the cystic lesion and the uterus (marked by arrows) 


\section{Cureus}

There was a strong suspicion of torsion and the patient was taken for surgery. On surgery, the cyst showed more than two twists around its pedicle (nearly $720^{*}$ ) and it also showed hemorrhagic congestion (Figure 5). The histopathology was that of cystadenoma with infarct changes (Figure 6).

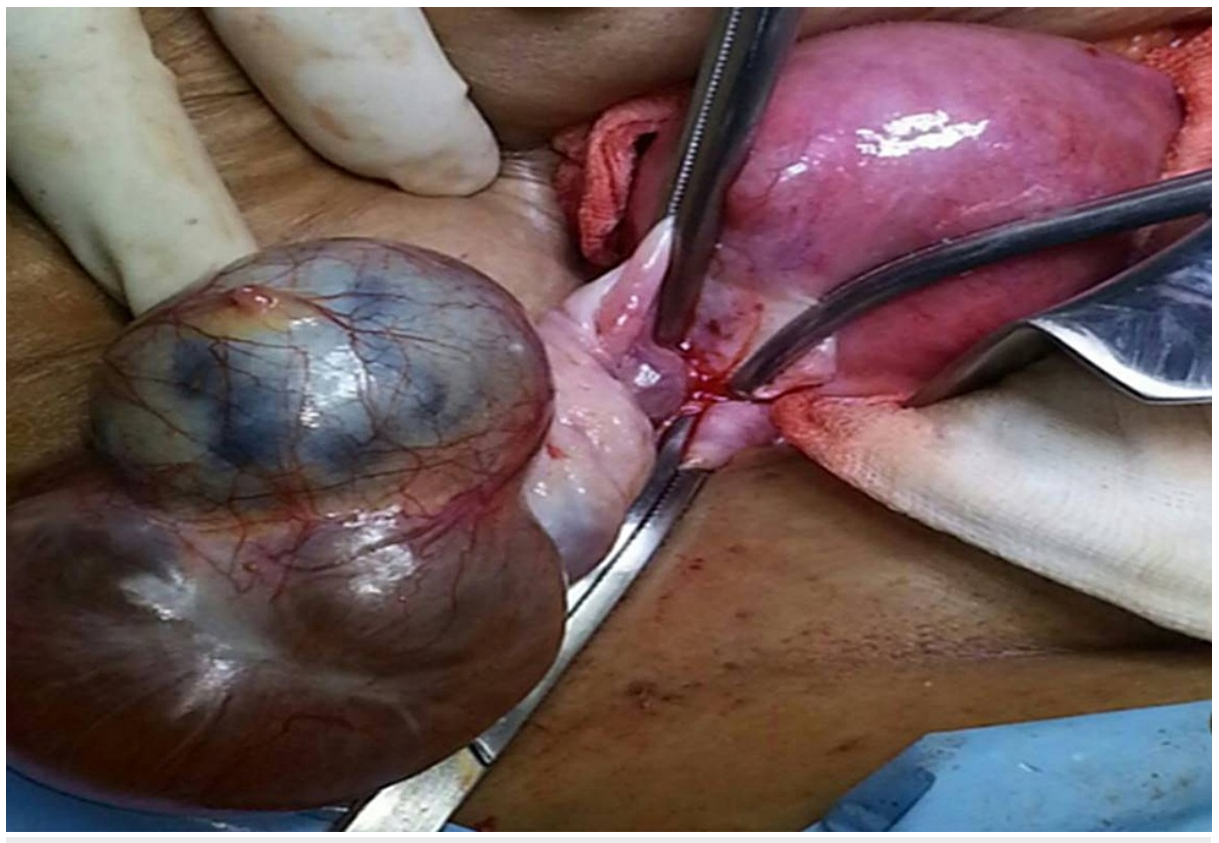

FIGURE 5: Case 2 Intra-operative photograph showing right ovarian cystic lesion with hemorrhagic infarction 


\section{Cureus}

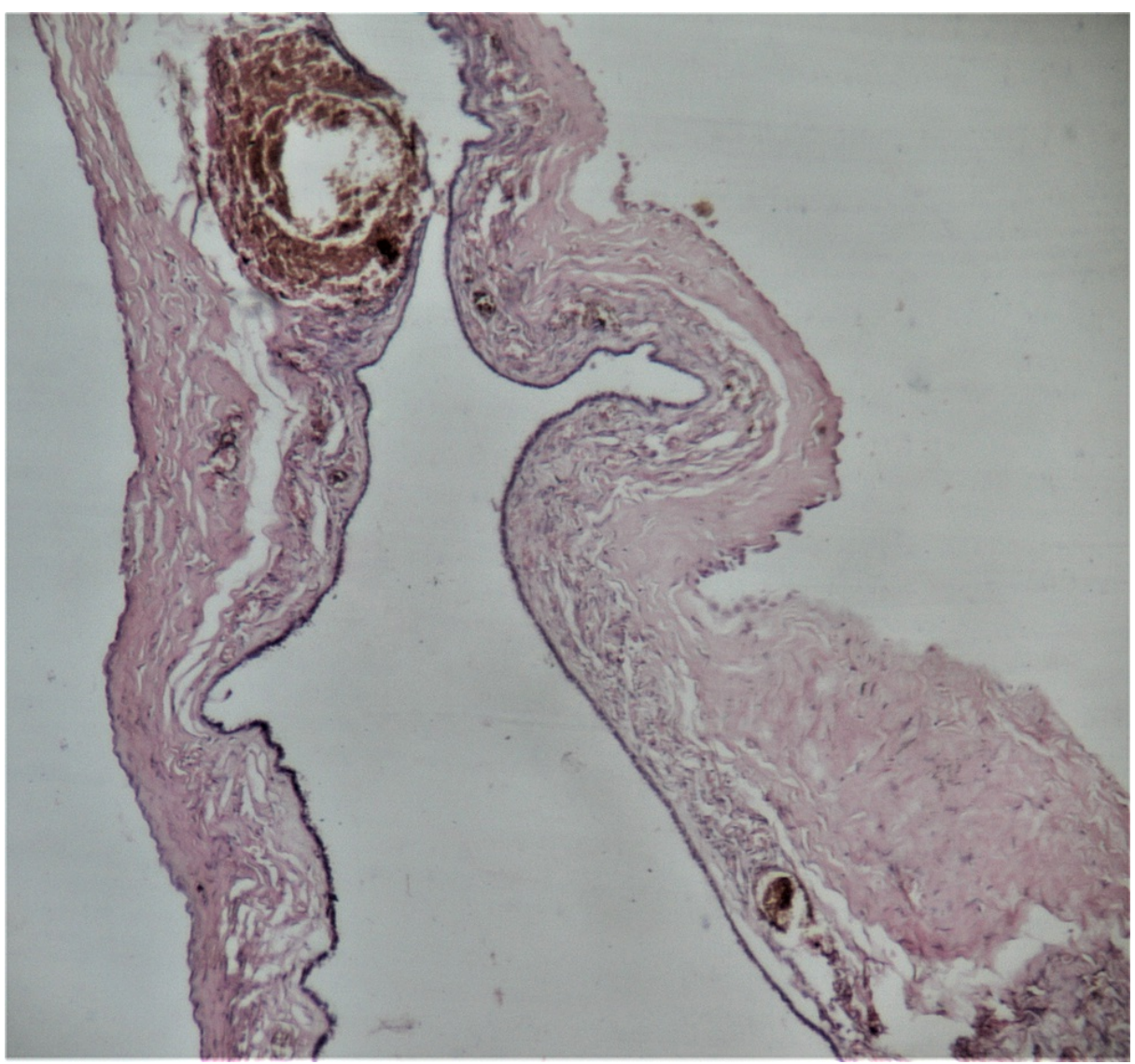

\section{FIGURE 6: Case 2 Histopathological section (H \& E stain; 4x magnification) showing ovarian cyst wall lined by ciliated cuboidal epithelium with focal denudation and perivascular hemorrhage around the congested blood vessel}

\section{Discussion}

Ovarian torsion is one of the acute gynecologic surgical emergencies that need early identification for proper management [5]. The first choice of evaluation may be a USG, but it is limited by operator dependence. The USG findings are abnormal location of the ovary, either anterior to or above the uterus or in the pouch of Douglas, ovarian edema, enlargement, ovarian cyst/mass, abnormal flow, and free fluid [6]. Other less common findings are tubal enlargement and hemorrhagic foci within the ovary. Another study mentioned the sonographic 'whirlpool' sign as a reliable sign of ovarian torsion [7]. However, the highest diagnostic accuracy achieved was $74.6 \%$ due to many factors, the primary being operator-dependence and the question of the need for further workup when the sonography is negative [6].

CT and MRI have been used in the evaluation of torsion in more subacute settings. As early as 1994, Kimura et al. [8] described CT and MRI findings of uterine deviation, engorged vessels, minimal free fluid, and obliteration of fat in cases of torsion. They also mentioned hemorrhagic cases (ovarian infarct), converging vessels from the ovary to the uterus, draped vessels around the lesion, areas of hemorrhage, and nonenhancement. Intravascular gas within an ovarian mass is a very specific, but extremely rare, finding of CT, and only one report has been described till now [9].

CT is routinely used as a problem-solving modality in acute abdomen that can also help to rule out torsion [10]. Rhe et al. [11] also studied CT and MRI findings and found that adnexal/tubal thickening and thickened smooth wall of the cystic lesion were seen in those who underwent hemorrhagic infarction. Duigenan et al. [12], in their study of CT/MRI findings with pathologic correlation, found that the twisted pedicle sign or helical swirling sign was pathognomonic and most specific of torsion, though it is slightly difficult to identify. A recent study by Mondoul et al. [13] compared the diagnostic performance of CT signs and found that inter-utero-ovarian mass was an independent, accurate, and reliable finding of torsion. The awareness of this specific sign may help both clinicians and radiologists to specifically look for this in cases of acute abdomen with lower abdominal pain. Swenson et al. [14] compared USG and CT in emergency settings and mentioned that when CT findings of torsion are present, another imaging modality like USG is not only time-consuming but also can delay the treatment. Both our cases were on the right side and torsion is 
reported more on the right side. The reason ascribed to this is colonic position on the left pelvis and hypermobility of the cecum/distal ileum on the right side [11].

Ito et al. [15] first described that the torsion angle determines the incidence of necrosis in torsion. They retrospectively studied CT findings of torsion and divided the patients based on the angle of torsion into less than $360 \%$ and more than $360 *$. The twisted pedicle with tube and mesovarium between the uterus and ovary was described as 'mass-like swelling'. They found that the twisted pedicle or mass-like swelling was seen mainly in those with torsion of more than $360 *$ and developed ovarian necrosis. This necrosis due to hemorrhagic infarction also had, in few cases, an area of high density due to hemorrhage and absent enhancement. In our first case, CT showed a multiloculated cyst on the right side of the uterus without any other changes. The second case showed a right-sided unilocular cystic lesion pushed above the right cornu of the uterus and showed thickened walls, the helical swirling sign of a twisted pedicle, and minimal free fluid. Surgically these correlated with less than 180 * twist in the first case and two rounds of twisting $(720 *)$ in the second. The first cyst showed only mild edematous swelling and the second lesion had changes of hemorrhagic infarction. It is to be seen by comparison of a larger number of patients with CT imaging whether this can be a reliable sign separating ovarian torsion with and without infarction or necrosis. Both of our patients were middle-aged and torsion is slightly less common at this age than in younger women. The reason is not known but it needs to be ascertained whether age-related ligamentous laxity or weakness could be a predisposing factor to torsion similar to its role in pelvic organ prolapse.

\section{Conclusions}

CT findings of twisted pedicle with pericystic fat stranding may be useful to predict those cases of adnexal torsion leading to infarction by indicating more than $360^{*}$ twisting of adnexal torsion and hence correlate with operative and gross findings of infarction in cases of torsion.

\section{Additional Information \\ Disclosures}

Human subjects: Consent was obtained or waived by all participants in this study. Conflicts of interest: In compliance with the ICMJE uniform disclosure form, all authors declare the following: Payment/services info: All authors have declared that no financial support was received from any organization for the submitted work. Financial relationships: All authors have declared that they have no financial relationships at present or within the previous three years with any organizations that might have an interest in the submitted work. Other relationships: All authors have declared that there are no other relationships or activities that could appear to have influenced the submitted work.

\section{References}

1. Lourenco AP, Swenson D, Tubbs RJ, Lazarus E: Ovarian and tubal torsion: imaging findings on US, CT, and MRI. Emerg Radiol. 2014, 21:179-87. 10.1007/s10140-013-1163-3

2. Chang HC, Bhatt S, Dogra VS: Pearls and pitfalls in diagnosis of ovarian torsion . Radiographics. 2008, 28:1355-68. 10.1148/rg.285075130

3. Houry D, Abbott JT: Ovarian torsion: a fifteen-year review . Ann Emerg Med. 2001, 38:156-9. 10.1067/mem.2001.114303

4. Comerci JT Jr, Licciardio F, Bergh PA, Gregori C, Breen JL: Mature cystic teratoma: a clinicopathologic evaluation of 517 cases and review of the literature. Obstet Gynecol. 1994, 84:22-8.

5. Ssi-Yan-Kai G, Rivain AL, Trichot C, Morcelet MC, Prevot S, Deffieux X, De Laveaucoupet J: What every radiologist should know about adnexal torsion. Emerg Radiol. 2018, 25:51-9. 10.1007/s10140-017-1549-8

6. Mashiach R, Melamed N, Gilad N, Ben-Shitrit G, Meizner I: Sonographic diagnosis of ovarian torsion: accuracy and predictive factors. J Ultrasound Med. 2011, 30:1205-10. 10.7863/jum.2011.30.9.1205

7. Vijayaraghavan SB: Sonographic whirlpool sign in ovarian torsion. J Ultrasound Med. 2004, 23:1643-51. 10.7863/jum.2004.23.12.1643

8. Kimura I, Togashi K, Kawakami S, Takakura K, Mori T, Konishi J: Ovarian torsion: CT and MR imaging appearances. Radiology. 1994, 190:337-41. 10.1148/radiology.190.2.8284378

9. Kawahara Y, Fukuda T, Futagawa S, Sakamoto I, Takao M, Kinoshita Y, Hayashi K: Intravascular gas within an ovarian tumor: a CT sign of ovarian torsion. J Comput Assist Tomogr. 1996, 20:154-6. 10.1097/00004728199601000-00028

10. Shyy W, Knight RS, Teismann NA: Right lower quadrant abdominal pain: do not forget about ovarian torsion on the computed tomography scan. J Emerg Med. 2018, 55:e43-5. 10.1016/j.jemermed.2018.01.006

11. Rha SE, Byun JY, Jung SE, et al.: CT and MR imaging features of adnexal torsion . Radiographics. 2002, 22:283-94. 10.1148/radiographics.22.2.g02mr02283

12. Duigenan S, Oliva E, Lee SI: Ovarian torsion: diagnostic features on CT and MRI with pathologic correlation . AJR Am J Roentgenol. 2012, 198:W122-31. 10.2214/AJR.10.7293

13. Mandoul C, Verheyden C, Curros-Doyon F, Rathat G, Taourel P, Millet I: Diagnostic performance of CT signs for predicting adnexal torsion in women presenting with an adnexal mass and abdominal pain: A casecontrol study. Eur J Radiol. 2018, 98:75-81. 10.1016/j.ejrad.2017.11.004

14. Swenson DW, Lourenco AP, Beaudoin FL, Grand DJ, Killelea AG, McGregor AJ: Ovarian torsion: case-control study comparing the sensitivity and specificity of ultrasonography and computed tomography for diagnosis in the emergency department. Eur J Radiol. 2014, 83:733-8. 10.1016/j.ejrad.2014.01.001

15. Ito K, Utano K, Kanazawa H, Sasaki T, Kijima S, Lefor AT, Sugimoto H: CT prediction of the degree of 


\section{Cureus}

ovarian torsion. Jpn J Radiol. 2015, 33:487-93. 10.1007/s11604-015-0452-z 